Research, Society and Development, v. 10, n. 3, e9910313084, 2021

(CC BY 4.0) | ISSN 2525-3409 | DOI: http://dx.doi.org/10.33448/rsd-v10i3.13084

\title{
Verificação empírica da escala de intenção a rotatividade: Validade de construto, de estrutura fatorial e invariância fatorial em trabalhadores
}

\author{
Empirical verification of the intent to turnover scale: Construct validity, factor structure and \\ factorial invariance in workers \\ Verificación empírica de la escala de intención de rotación: Validez de constructo, estructura \\ factorial e invarianza factorial en trabajadores
}

Recebido: 18/02/2021 | Revisado: 26/02/2021 | Aceito: 01/03/2021 | Publicado: 07/03/2021

\author{
Nilton Soares Formiga \\ ORCID: https://orcid.org/0000-0003-4907-9736 \\ Universidade Potiguar, Brasil \\ E-mail: nsformiga@yahoo.com \\ Fernando Santos do Nascimento \\ ORCID: https://orcid.org/ 0000-0002-7826-153X \\ Universidade Potiguar, Brasil \\ E-mail: nsformiga@yahoo.com \\ Juliana Bianca Maia Franco \\ ORCID: http://orcid.org/0000-0002-7566-2688 \\ Universidade Potiguar, Brasil \\ E-mail: juliana_franco_@hotmail.com \\ Heitor César Costa Oliveira \\ ORCID: https://orcid.org/0000-0003-4778-6613 \\ Universidade Potiguar, Brasil \\ E-mail: cartoriosbrasil@gmail.com \\ Cristienne Gonçalves Pereira Valin \\ ORCID: http://orcid.org/0000-0002-7928-4856 \\ Universidade Potiguar, Brasil \\ E-mail: cris2n@hotmail.com \\ Gleyssielle Lira Prochazka \\ ORCID: https://orcid.org/0000-0001-6083-1306 \\ Universidade Potiguar, Brasil \\ E-mail: gleyssielle.lira@gmail.com \\ Ricardo Ovídio de Oliveira Lima \\ ORCID: https:// orcid.org/0000-0002-1645-7537 \\ Universidade Potiguar, Brasil \\ E-mail: adv@hotmail.com \\ Thalita Kelle Pires Beserra \\ ORCID: https:// orcid.org/0000-0003-2693-8702 \\ Universidade Potiguar, Brasil \\ E-mail: tpiresb@gmail.com \\ Ennio Alves de Sousa Andrade Lima \\ ORCID: https://orcid.org/0000-0002-2259-2532 \\ Universidade Potiguar, Brasil \\ E-mail: unicoennio@hotmail.com
}

\section{Resumo}

Este artigo tem como objetivo verificar a qualidade da estrutura fatorial, invariância e diferenças da escala de intenção a rotatividade em trabalhadores. O tema a respeito da rotatividade no ambiente de trabalho tem sido estudado com frequência nos últimos anos, o qual, é uma preocupação por parte de trabalhadores e gestores, pois, afeta diretamente na qualidade e eficiência das organizações, capaz de comprometer as estratégias de competitividade, produtividade e inovação. Participaram do estudo, 219 trabalhadores, com idades acima de 18 anos, distribuídos a cidade de Natal-RN e João Pessoa-PB, no setor público e privado, homens e mulheres. Estes responderam, além dos dados sociodemográficos, as escalas de intenção a rotatividade, Escala de Percepção de Suporte Organizacional e a Escala de percepção de assédio moral no mundo do trabalho. Realizaram-se as estatísticas descritivas, correlações de Pearson, teste t de Student, Anova e a análise fatorial confirmatória. A parti dos achados neste estudo, destaca-se que a referida escala é confiável, consiste e invariável, especialmente, quando compararam as amostras do RN e PB. Os indicadores psicométricos tanto corresponderam ao que era esperado quanto esteve, tecnicamente, inserido nos 
critérios estatísticos exigidos. Com isso, considera-se que esta medida se destina ao uso no contexto organizacional, sendo importante para à avaliação do recurso humano nas organizações, com foco tanto no diagnóstico quanto prognóstico laboral.

Palavras-chave: Rotatividade; Escala; Construto; Análise fatorial.

\begin{abstract}
This paper aims to verify the quality of factor structure, invariance and differences of the turnover intention scale in workers. The theme regarding turnover in the workplace has been studied frequently in recent years, which is a concern for workers and managers, because it directly affects the quality and efficiency of organizations, capable of compromising the strategies of competitiveness, productivity and innovation. The study had the participation of 219 workers, aged over 18 years old, distributed in the cities of Natal-RN and João Pessoa-PB, in the public and private sectors, men and women. They answered, besides the sociodemographic data, the Turnover Intention Scale, the Survey of Perceived Organizational Support, and the Scale of Perception of Bullying in the World of Work. Descriptive statistics, Pearson correlations, Student's t-test, Anova, and confirmatory factorial analysis were performed. From the findings of this study, the scale is reliable, consistent, and invariable, especially when comparing the RN and PB samples. The psychometric indicators both corresponded to what was expected and were technically within the required statistical criteria. Thus, it is considered that this measure is intended for use in the organizational context, being important for the evaluation of human resources in organizations, focusing both on diagnosis and labor prognosis.
\end{abstract}

Keywords: Turnover; Scale; Construct; Factor analysis.

\title{
Resumen
}

Este artículo tiene como objetivo verificar la calidad de la estructura factorial, invarianza y diferencias en la escala de intención de rotación en trabajadores. El tema de la rotación en el lugar de trabajo se ha estudiado con frecuencia en los últimos años, lo que preocupa a trabajadores y directivos, ya que afecta directamente a la calidad y eficiencia de las organizaciones, capaces de comprometer las estrategias de competitividad, productividad e innovación. En el estudio participaron 219 trabajadores, mayores de 18 años, distribuidos en la ciudad de Natal-RN y João Pessoa-PB, del sector público y privado, hombres y mujeres. Estos respondieron, además de los datos sociodemográficos, las escalas de intención de recambio, la Escala de Percepción de Apoyo Organizacional y la Escala de percepción del acoso en el mundo laboral. Se realizó estadística descriptiva, correlaciones de Pearson, t de Student, ANOVA y análisis factorial confirmatorio. Con base en los hallazgos de este estudio, se destaca que la escala referida es confiable, consistente e invariable, especialmente al comparar las muestras de NB y BP. Los indicadores psicométricos correspondieron a lo esperado y, técnicamente, se incluyeron en los criterios estadísticos requeridos. Así, se considera que esta medida está pensada para su uso en el contexto organizacional, siendo importante para la evaluación de los recursos humanos en las organizaciones, enfocándose tanto en el diagnóstico como en el pronóstico laboral.

Palabras clave: Rotación; Escala; Construcción; Análisis factorial.

\section{Introdução}

A intenção de rotatividade, a rotatividade e a retenção de pessoal são consideradas temas centrais de preocupação de trabalhadores e gestores nos últimos dez anos; esse fato não se deve apenas a iniciação dos novos talentos, mas, também, aos gastos que podem gerar para as instituições e recursos humanos quanto ao recrutamento, seleção e treinamento de pessoal (Stell, Penha, \& Bonilla, 2016; Silva, 2018).

Tais condições afetam diretamente na qualidade e eficiência das organizações, visto que provocam perda de talentos profissionais competentes e produtivos, além de comprometer as estratégias de competitividade, produtividade e inovação (Mintzberg \& Lampel, 1999; Siqueira, Gomide, Oliveira, \& Polizzi, 2014; Agapito, 2012; Agapito, Polizzi, \& Siqueira, 2015).

As condições atuais do mercado de trabalho têm influenciado a qualificação profissional e dos serviços prestados pelas organizações, tanto no que se refere às demandas funcionais, quanto à estrutura nas organizações. Com isso, as organizações têm investido em estratégias de recrutamento na seleção e admissão de colaboradores que sejam competentes em sua especialidade, mas também, em suas emoções e relações interpessoais (cf. Pereira, Formiga, \& Estavam, 2019).

O investimento direto nas estratégias de recrutamento, seleção e treinamento de pessoal tem como foco uma melhor filtragem, não apenas de pessoas com um currículo competitivo, mas também com maior capital humano e intelectual, 
Research, Society and Development, v. 10, n. 3, e9910313084, 2021

(CC BY 4.0) | ISSN 2525-3409 | DOI: http://dx.doi.org/10.33448/rsd-v10i3.13084

observados cada vez mais nas políticas de desenvolvimento e treinamento para as empresas em geral (Pontes, 1996; Fleury \& Fleury, 2000; Albuquerque, 2002; Faissal, Passos, Mendonça, et al., 2009).

Frente ao fenômeno da rotatividade nas organizações, tem se cogitado que as empresas se concentrem mais nas estratégias de retenção de pessoal, no que se refere ao capital humano, do que exclusivamente, nas questões econômicas; com colaboradores comprometidos, engajados e confiantes, provoca-se nos gestores, diretores e/ ou empresários maior atração para o desenvolvimento, treinamento e retenção de colaboradores que contribuam para os objetivos e metas organizacionais (Mobley, 1992; Borges-Andrade, Abbad, \& Mourão, 2005; Joo \& McLean, 2006; Silveira, 2011; Oliveira, Gomide, Sinésio, Bânia, \& Oliveira-Silva, 2018).

Provavelmente a existência e sobrevivência das empresas estariam, ao considerar as reflexões dos autores supracitados, associadas a este único diferencial: o capital pessoal que estas possuem; condição esta que sugere o desenvolvimento de estratégias que proporcionem aos colaboradores condições positivas em direção a 'um olhar para o futuro', facilitando negócios rentáveis e competitivos para a organização ao estruturar e alicerçar uma força de trabalho otimizada em capacidades cognitivas e comportamentais organizacionais (Ulrich, 1998; Formiga, Freire, Azevedo, \& Faria, 2020).

Para isso, é de extrema importância tanto a quantificação do valor dos recursos humanos por parte das empresas considerando o fato de que as pessoas não devem ser vistas apenas como recursos a serem consumidos e de fácil reposiçãoquanto o apoio de criação e desenvolvimento de vínculo organizacional gerido pela empresa aos seus colaboradores, fatos estes que seriam capazes de permitir que organização-trabalhador reconheçam que são partes mútuas para a qualidade e sustentabilidade dos processos organizacionais e de recursos humanos (Brian, Hatch, \& Walker, 2000; Dalfovo, \& Dias, 2009).

Inibir o processo da rotatividade requer investir em políticas organizacionais para retenção de talentos; esta poderá ser definida como uma espécie de esforço despendido por uma organização para manter os talentos satisfeitos e comprometidos com seus resultados, gerando motivação para nela permanecerem. Com isso, a retenção de talentos seria o sucesso que uma organização pode ter devido a uma estrutura e funcionalidade muito mais preocupada em uma gestão humana e valorada com vistas a um quadro funcional destinado ao incentivo e manutenção da empresa e colaboradores competitivos, os quais estão sempre atentos a um mundo em rápidas e constantes mudanças sociais, econômicas e laborais (Mendonça, 2002; Tamayo \& Paschoal, 2003; Yamashita, 2007).

De fato, apesar da existência de estudiosos que consideram a rotatividade uma espécie de movimento organizacional que contribuiria para uma melhor filtragem de talentos e políticas funcionais laborais (cf. Siqueira, Gomide, Oliveira, \& Polizzi, 2014), existe um custo econômico deste fenômeno, entre eles: há o custo de separação, contabilizado por fatores relacionados à saída do empregado da organização, por exemplo, as entrevistas de desligamento, custos legais, etc.; o custo de recolocação de pessoal, com vistas a um envolvimento de ações relacionadas ao recrutamento e seleção de pessoal; e o custo de treinamento, que contempla as estratégias de redução do tempo de adaptação funcional à cultura da organização, bem como, o treinamento associado ao desenvolvimento de suas atividades (Schwab, 1991; Homn, 1992).

Dado o impacto que a rotatividade ocasiona nas empresas e no recurso humano, esta pesquisa tem como objetivo principal verificar a qualidade psicométrica de uma medida de intenção a rotatividade, a qual, desenvolvida por Siqueira et al. (1997), revelando indicadores psicométricos confiáveis em trabalhadores; bem como, pretende-se avaliar a validade convergente e divergente (respectivamente, associada ao suporte organizacional e assédio moral no mundo do trabalho), buscando compreender as causas e consequências que identifiquem os custos objetivos e subjetivos deste fenômeno na organização 
Research, Society and Development, v. 10, n. 3, e9910313084, 2021

(CC BY 4.0) | ISSN 2525-3409 | DOI: http://dx.doi.org/10.33448/rsd-v10i3.13084

\section{Metodologia}

\section{Amostra}

Abordou-se um descritivo, exploratório e correlacional, de perspectiva quantitativa focado em trabalhadores de organizações públicas e privadas; este estudo foi do tipo não probabilística e intencional com o ' $\mathrm{n}$ ' amostral avaliado no através do pacote estatístico GPower 3.1 (Faul, Erdfelder, Lang, \& Buchner, 2007). Para a coleta de dados estabeleceu uma probabilidade de $95 \%$ ( $\mathrm{p}<0,05)$, magnitude do efeito amostral $(r \geq 0,50)$ e um padrão de poder hipotético $(\pi \geq 0,80)$, com os quais, revelou que uma amostra de 219 trabalhadores, apresentou indicadores estatísticos $(t \geq 1,98 ; \pi=0,95 ; \mathrm{p}<0,01)$ que garantiram a qualidade da amostra, tendo as seguintes características sociodemográficas: idades acima de 18 anos, distribuídos a cidade de Natal-RN e João Pessoa-PB, nos setores público (38\%), privado (47\%) e com participantes trabalhando em ambos os setores (15\%); em relação ao sexo, observou-se que as mulheres foram a maioria (66\%) e homens (34\%) e quanto ao estado civil, $44 \%$ eram casados, $42 \%$ solteiro, $9 \%$ tem união estável, $5 \%$ divorciados e 1\% viúvo. Em termos de temporalidade laboral, esses profissionais, tinham um tempo de serviço que variou de 1 a 42 anos $(M=9,19$, d.p. = 8,19). Em relação, a nível de educação, $36 \%$ tinham especialização, $28 \%$ superior, $15 \%$ nível médio, $13 \%$ mestrado e 5\% doutorado.

\section{Instrumentos}

Os sujeitos responderam a um questionário que continha dados sociodemográficos (idade, sexo, renda econômica) e laboral (por exemplo, tempo de serviço, tipo de organização) e, as seguintes escalas:

Escala de Intenção a Rotatividade (EIR) - Medida desenvolvida por Siqueira et al. (1997), composta por três itens (por exemplo, você pensa em sair da organização onde trabalha; Você planeja sair da organização onde trabalha; Você tem vontade de sair da organização onde trabalha), para os quais, o respondente deverá indicar numa escala do tipo Likert de cinco pontos, variando de 1 (nunca) a 5 (sempre), o quanto está de acordo ou não, com as informações expressas no itens, a qual apresentou um índice de precisão acima de $0,70(\alpha=0,95)$ e por contemplar uma definição clara e aplicada para a avaliação do fenômeno pretendido no espaço laboral.

Escala de Percepção de Suporte Organizacional (EPSO) - Escala desenvolvida por Eisenberger et al. (1986), adaptada e validade para o contexto brasileiro por Siqueira (1995), sendo composta por 9 itens (SO1 = Esta empresa ignoraria qualquer reclamação de minha parte; $\mathrm{SO} 2$ = Esta empresa não considera meus interesses quando toma decisões que me afetam; $\mathrm{SO} 3=\mathrm{E}$ possível obter ajuda desta empresa quando tenho um problema; SO4 = Esta empresa realmente preocupa-se com meu bemestar; SO5 = Esta empresa estaria disposta a ampliar suas instalações para me ajudar a utilizar minhas melhores habilidades no desempenho do meu trabalho; SO6 = Esta empresa está pronta a ajudar-me quando eu precisar de um favor especial; SO7 = Esta empresa preocupa-se com minha satisfação com o trabalho; SO8 = Esta empresa preocupa-se mais com seus lucros do que comigo e SO9 = Esta empresa tenta fazer com que meu trabalho seja o mais interessante possível).

O objetivo principal da escala é o de avaliar o quanto os trabalhadores percebem que a empresa tem uma preocupação com o bem-estar do empregado. Para mensurar o construto, o respondente deveria indicar a sua resposta, marcando com um $\mathrm{X}$ ou um círculo, em uma escala de sete pontos que variava de $1=$ discordo totalmente a $7=$ concordo totalmente. No pioneiro estudo de Siqueira (1995), essa medida apresentou um alfa de 0,86, revelando uma consistência interna. Numa com trabalhadores brasileiros, Formiga, Fleury e Souza (2014), através da análise fatorial confirmatória, observou que a consistência da estrutura fatorial da escala permanece, tendo apresentado os seguintes indicadores psicométricos $\left(X^{2} / \mathrm{gl}=1,42\right.$, $\mathrm{RMR}=0,02, \mathrm{GFI}=0,99, \mathrm{AGFI}=0,97, \mathrm{CFI}=0,99, \mathrm{TLI}=0,99$ e $\mathrm{RMSEA}=0,03)$.

Escala de percepção de assédio moral no mundo do trabalho (EPAMT): trata-se de um instrumento composto por 24 itens (questões), o qual, foi desenvolvido por Martins e Ferraz (2014); os itens destacam os atos de violência praticados no 
Research, Society and Development, v. 10, n. 3, e9910313084, 2021

(CC BY 4.0) | ISSN 2525-3409 | DOI: http://dx.doi.org/10.33448/rsd-v10i3.13084

trabalho pelo chefe e/ou subordinados, tendo como objetivo, agredir, de forma sutil ou direta, o trabalhador nos aspectos da sua profissão ou vida pessoal. O respondente deveria indicar sua resposta numa escala do tipo Likert, com variação que salienta à frequência da ocorrência de determinada situação (por exemplo, 1- nunca ou quase nunca; 2 - menos de uma vez ao mês; 3 ao menos uma vez ao mês; 4 - mais de uma vez ao mês; 5 - ao menos uma vez por semana; 6 - várias vezes por semana e 7 uma ou mais vezes ao dia).

No estudo de Lima, Lima e Formiga (2019), com trabalhadores do setor público da saúde e educação, os indicadores psicométricos confiáveis, revelando alfas acima de 0,70 e CC e VME, respectivamente, superior a 0,80 e 0,50, corroborando a avaliação teórica e empírica do assédio moral em ambos os setores de trabalho.

\section{Procedimentos e administração do instrumento}

Colaboradores com experiência prévia na administração dos instrumentos foram responsabilizados pela coleta dos dados, e apresentaram-se in loco ou por meio de questionários eletrônicos inseridos na plataforma de acesso google forms, os quais eram enviados por e-mail e compartilhado em redes sociais. Para isso, informava-lhes que os instrumentos se referiam ao conhecimento das opiniões e os comportamentos sobre as situações descritas relativa aos trabalhadores em distintas empresas.

Solicitou-se a colaboração voluntária das pessoas no sentido de responderem um breve questionário. Após ficarem cientes das condições de participação na pesquisa, assinaram um termo de Consentimento Livre e Esclarecido. Foi-lhes dito que não havia resposta certa ou errada. A todos foi assegurado o anonimato das suas respostas informando que estas seriam tratadas em seu conjunto. O questionário foi respondido individualmente quando in loco em ambas as cidades, bem como, na forma de compartilhamento eletrônico nas redes sociais e de mensagens (Facebook, Instagram e WhataApp), ou ainda via email; nestes, o instrumento foi hospedado e disponibilizado via doc.google.com/forms.

Apesar de o instrumento ser auto-aplicável, contando com as instruções necessárias para que possam ser respondidos, os colaboradores estiveram presentes durante toda a aplicação, quando em local físico, e por e-mail ou rede social, quando no meio eletrônico, para retirar eventuais dúvidas ou realizar esclarecimentos que se fizessem indispensáveis. Um tempo médio de 40 minutos foi suficiente para concluir essa atividade.

Todos os procedimentos adotados nesta pesquisa seguiram as orientações previstas na Resolução 466/2012 do CNS e na Resolução 016/2000 do Conselho Federal de Psicologia para as pesquisas com seres humanos (Conselho Nacional de Saúde [CNS], 2012; ANPEPP, 2000).

\section{Análise dos dados}

Quanto à análise dos dados, realizou-se, além das análises descritivas, correlações de Pearson, teste t de Student, Anova, etc.; efetuou-se também, uma análise fatorial confirmatória a fim de avaliar a estrutura fatorial da escala de intenção a rotatividade. Considerou-se como entrada a matriz de covariâncias o estimador ML (Maximum Likelihood). Sendo um tipo de análise estatística mais criteriosa e rigorosa, buscou-se avaliar a estrutura teórica do DASS-21 com quatro fatores. A seguir serão apresentados esses indicadores:

$\mathrm{O} \chi^{2}$ (qui-quadrado) testa a probabilidade do modelo teórico se ajustar aos dados: quanto maior o valor do $\chi^{2}$ pior o ajustamento. Entretanto, ele tem sido pouco empregado na literatura, sendo mais comum considerar sua razão em relação aos graus de liberdade ( $\chi^{2} / g .1$.). Neste caso, valores até 3 indicam um ajustamento adequado; Raiz Quadrada Média Residual (RMR), que indica o ajustamento do modelo teórico aos dados, na medida em que a diferença entre os dois se aproxima de zero; O Goodness-of-Fit Index (GFI) e o Adjusted Goodness-of-Fit Index (AGFI) são análogos ao $R^{2}$ na regressão múltipla e, portanto, indicam a proporção de variância-covariância nos dados explicada pelo modelo. Os valores desses indicadores 
variam de 0 a 1 , sendo que os valores na casa dos 0,80 e 0,90, ou superiores, indicam um ajustamento satisfatório; A RootMean-Square Error of Approximation (RMSEA), com seu intervalo de confiança de 90\% (IC90\%), é considerado um indicador de "maldade" de ajuste, isto é, valores altos indicam um modelo não ajustado. Assume-se como ideal que o RMSEA se situe entre 0,05 e 0,08, aceitando-se valores até 0,10; O Comparative Fit Index (CFI) - compara de forma geral o modelo estimado ao modelo nulo, considerando valores mais próximos de um como indicadores de ajustamento satisfatório; O Expected CrossValidation Index (ECVI) e o Consistent Akaike Information Criterion (CAIC) são indicadores geralmente empregados para avaliar a adequação de um modelo determinado em relação a outro. Valores baixos do ECVI e CAIC expressam o modelo com melhor ajuste (Byrne, 1989; Joreskög \& Sörbom, 1989; Hair, Anderson, Tatham \& Black, 2005).

Realizaram-se, tanto o cálculo de confiabilidade composta (CC) quanto da variância média extraída (VME); no primeiro indicador exige-se que o nível do escore seja acima de 0,70, enquanto no segundo indicador é preciso um nível acima de 0,50 (Hair, Anderson, Tatham \& Black, 2005). Bem como, correlação de Pearson, teste t de Student e Anova one-way.

\section{Resultados e Discussão}

Os dados constituintes da amostra foram inseridos na planilha do SPSS for Windows, e realizaram-se análises estatísticas para avaliar a normalidade da amostra coletada: no que diz respeito aos dados omissos do estudo, eles não foram acima de 5\%, definido por Tabachnick e Fidell (2001); quanto a multicolinearidade entre as variáveis, as correlações entre elas estiveram dentro dos parâmetros definidos por Tabachnick e Fidell (2001) [r $\leq$ 0,90,], pois elas variaram de 0,05 a 0,86. Em relação a presença de outliers multivariados na amostra, com base no teste de normalidade de Shapiro-Wilk (S-W), observouse uma normalidade $(\mathrm{S}-\mathrm{W}=0,83)$ da amostra a um $\mathrm{p}<0,14$ (cf. Nascimento, Tibana, Melo, \& Prestes, 2015).

Identificada a normalidade da amostra, viu-se a necessidade de avaliar a convergência da perspectiva teórica e empírica do conteúdo dos itens na referida escala; para isso, primeiro, verificou-se a discriminação dos itens, em seguida, a relação entre os termos dos itens e sua representatividade comportamento-domínio. Para realização do primeiro cálculo, avaliou-se o poder discriminativo e a representatividade dos itens (ver tabela 1) para a EIRT.

Tais cálculos tem como objetivo apresentar maior especificidade estatística destinada a organização e verificação da distribuição de respostas na medida utilizada. Orientado pelos pressupostos da Teoria Clássica dos Testes (TCT) (Formiga, Fleury, Fandiño, \& Souza, 2016), bem como, nas direções axiomáticas observadas na fatorialização da EIRT no estudo de Siqueira, Gomide, Oliveira e Polizzi (2014) procurou-se observar o quanto cada um dos itens (IRT 1 - Você pensa em sair da empresa onde trabalha [refere-se à avaliação do pensamento]; IRT 2 - Você planeja sair da empresa onde trabalha [diz respeito à avaliação do planejamento]; IRT 3 - Você tem vontade de sair da empresa onde trabalha [considera à avaliação da volição ou vontade]) foi capaz de discriminar as respostas das pessoas com magnitudes próximas, isto é, se discriminam os grupos inferiores e superiores com relação ao construto mensurado.

Para atender essa condição hipotético-metodológica (a discriminação dos itens das escalas), foi calculada uma pontuação total de cada escala e em seguida a sua mediana ( $\mathrm{Me}=9,00$; $\operatorname{Min}=3,00$ e $\mathrm{Max}=15,00)$; aqueles respondentes que apresentassem pontuação abaixo da mediana foram classificados como um grupo inferior, enquanto aqueles com pontuações acima da mediana formaram o grupo superior (cf. Formiga, Fleury, Fandiño, \& Souza, 2016). Considerando-se cada um dos itens do IRT, efetuou-se um teste t para amostras independentes e compararam-se ambos os grupos observando quais deles discriminam as pessoas com magnitudes próximas e estatisticamente significativas (tabela 1).

$\mathrm{Na}$ Tabela 1, destaca-se que todos os itens, em sua especificidade, discriminam as pontuações dos sujeitos em cada no IRT. Sendo assim, estes itens, na indicação dos respondentes, quanto maior ou menor for a pontuação expressa na escala, revela que na escala de resposta, a compreensão do conteúdo estabelecido por Siqueira, Gomide, Oliveira e Polizzi (2014), foi 
garantida pelos respondentes. Ainda na mesma tabela, tratou-se de verificar a relação entre os itens e o construto, o qual tem como base a representatividade comportamento-domínio.

Com isso, sistematicamente, avaliou-se a relação teórica do teste e as situações especificadas nos itens e o quanto eles representam os aspectos esperados (cf. Formiga, Fleury, Fandiño, \& Souza, 2016). A partir do cálculo da correlação de Pearson (r), o qual verifica a relação dos itens da escala com a pontuação total da mesma, condição essa que se espera existir uma correlação positiva acima de 0,50 e significativa. Observou-se na Tabela 1, que a correlação (r) itens-construto da EIRT, foram positivas, significativas e acima de escore correlacional exigido, sendo capaz de representar, significativamente o binômio comportamento-domínio relacionado ao construto proposto.

Tabela 1: Poder discriminativo e representatividade de conteúdo dos itens da EIRT.

\begin{tabular}{|c|c|c|c|c|}
\hline Construtos & \multirow[t]{2}{*}{ Itens } & \multicolumn{3}{|c|}{ Estatística } \\
\hline \multirow{4}{*}{ 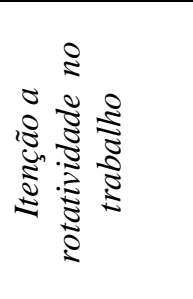 } & & $t$ & $\mathrm{r}_{\text {item-fator }}$ & p-valor \\
\hline & $\begin{array}{l}\text { IRT } 1 \text { - Você pensa em sair da empresa } \\
\text { onde trabalha. }\end{array}$ & $-2,47$ & 0,90 & 0,001 \\
\hline & $\begin{array}{l}\text { IRT } 2 \text { - Você planeja sair da empresa } \\
\text { onde trabalha. }\end{array}$ & $-2,62$ & 0,88 & 0,001 \\
\hline & $\begin{array}{l}\text { IRT } 3 \text { - Você tem vontade de sair da } \\
\text { empresa onde trabalha. }\end{array}$ & $-2,65$ & 0,96 & 0,001 \\
\hline
\end{tabular}

Fonte: Autores.

A título de acréscimo, na avaliação do conteúdo dos itens da EIRT, devido a qualidade dos resultados na discriminação e representatividade dos itens, utilizou-se o método de Lawshe ou Conteúdo de Validade Crítica (em inglês,

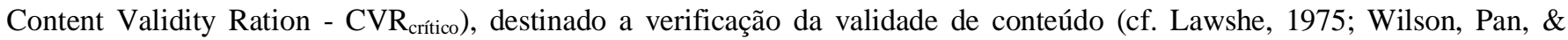
Schumsky, 2012).

Para isso, especialistas na área da Psicologia Organizacional e do Trabalho avaliaram o quanto os itens (IRT 1 - Você pensa em sair da empresa onde trabalha; IRT 2 - Você planeja sair da empresa onde trabalha; IRT 3 - Você tem vontade de sair da empresa onde trabalha) seriam de essencial importância na avaliação da intenção a rotatividade. Observou-se a existência de que em todos os três itens houve um percentual acima de 50\% na validade do conteúdo expressos pelos juízes, a saber: IRT $1=$ $89 \%$, IRT $2=92 \%$ e IRT $3=94 \%$. Tais resultados não apenas corroboram a qualidade da manutenção conteúdo-construto do item, previamente observado numa amostra de trabalhadores, mas também que os próprios especialistas na área da Psicologia Organizacional e do Trabalho estão em acordo quanto aos itens e o que eles expressam para avaliar a intenção a rotatividade.

Ainda quanto a um objetivo adicional de consistência interna, efetuou o cálculo do coeficiente do Lambda $(\lambda) 2$ de Guttman; de acordo com Formiga, Souza, Costa, Gomes, Fleury e Melo (2015), o uso de tal coeficiente diz respeito ao fato de que, em alguns estudos, ele é um indicador de melhor estimativa de confiabilidade quanto o instrumento avaliado e composto por poucos itens. Observou-se a existência de Lambda $(\lambda)$ com escores satisfatórios, acima de 0,70 $(\lambda=0,96)$. Estes achados, revelaram a fidedignidade da medida de intenção a rotatividade de trabalhadores com amostra em dois contextos distintos (João Pessoa-PB e Natal-RN); nestas o Lambda foi também próximo ao observado na amostra total (respectivamente, 0,94 e $0,92)$.

A partir desses resultados, os quais, revelaram que os itens, tanto discriminam quanto representam a relação itemconstruto do conteúdo da EIRT, verificou-se a estrutura fatorial considerando os achados Siqueira, Junior, Oliveira e Pollizi (2014) relativos a EIRT. Nesta etapa, focou-se nos referidos cálculos da TCT e, também, na proposta axiomática que orientou de forma representacional o construto reflexivo do modelo estrutural com um único fator. Com o objetivo de garantir a 
qualidade da medida em questão, gerou-se tanto o modelo proposto (estrutura unifatorial ajustada) quanto modelo comparativo (isto é, estrutura unifatorial não ajustada). Os resultados revelaram que o modelo unifatorial ajustado apresentou melhores indicadores psicométricos (ver Tabela 2).

Tabela 2: Indicadores psicométricos da comparação da estrutura fatorial do EIRT.

\begin{tabular}{|c|c|c|c|c|c|c|c|c|c|}
\hline \multirow{2}{*}{ Modelos } & \multicolumn{4}{|c|}{ Medidas de ajuste absoluto } & \multicolumn{3}{|c|}{ Medidas de ajuste incremental } & \multicolumn{2}{|c|}{$\begin{array}{l}\text { Medidas de ajuste } \\
\text { parcimonioso }\end{array}$} \\
\hline & $\chi^{2} / g \mathbf{g l}$ & RMR & GFI & AGFI & CFI & TLI & $\begin{array}{c}\text { RMSEA } \\
\text { (intervalo) }\end{array}$ & CAIC & $\begin{array}{c}\text { ECVI } \\
\text { (intervalo) }\end{array}$ \\
\hline $\begin{array}{l}\text { Unifatorial } \\
\text { não ajustado }\end{array}$ & 3,25 & 0,10 & 0,90 & 0,88 & 0,91 & 0,93 & $\begin{array}{c}0,06 \\
(0,05-0,09\end{array}$ & 95,78 & $\begin{array}{c}1,05 \\
(1,01-1,86)\end{array}$ \\
\hline $\begin{array}{l}\text { Unifatorial } \\
\text { ajustado }\end{array}$ & 1,57 & 0,01 & 0,99 & 0,99 & 1,00 & 1,00 & $\begin{array}{c}0,01 \\
(0,00-0,02)\end{array}$ & 52,22 & $\begin{array}{c}0,08 \\
(0,06-0,12\end{array}$ \\
\hline
\end{tabular}

Fonte: Autores.

Outros indicadores estatísticos, com uma tendência à avaliação parcimoniosa (por exemplo, o AIC, BIC e BCC) do modelo fatorial pretendido garantiram a proposta unifatorial hipotetizada; estes indicadores, acompanham o CAIC e ECVI, os quais destinados a verificação da adequabilidade pretenso modelo, especialmente quando se trata da comparação entre estruturas fatoriais (cf. Marôco, 2010). Como observado na Tabela 3, os melhores indicadores estiveram para o modelo unifatorial ajustado.

Tabela 3: Indicadores psicométricos de parcimônia para a comparação da estrutura fatorial da EIRT

\begin{tabular}{cccc}
\hline Modelos & \multicolumn{3}{c}{ Indicadores de Parcimônia } \\
\cline { 2 - 4 } & AIC & BIC & BCC \\
\hline Unifatorial não ajustado & 35,69 & 68,52 & 33,26 \\
\hline Unifatorial ajustado & 17,11 & 44,22 & 17,49 \\
\hline
\end{tabular}

Fonte: Autores.

Na Tabela 4 é possível observar que todas as saturações (Lambdas, $\lambda$ ) estão no intervalo esperado $\mid 0$ - $1 \mid$, permitindo afirmar a inexistência de problemas de estimação proposta; além de estarem inseridas nestes parâmetros, foram estatisticamente diferentes de zero $(t>1,96, p<0,05)$, garantindo a qualidade da validade da estrutura fatorial do EIRT.

Destaca-se também a validade do construto em questão, esta, foi observada com base no cálculo de confiabilidade composta (CC), tendo como critério escore acima de 0,70 e da variância média extraída (VME) que deverá apresentar um escore acima de 0,50. Desta forma, observou-se que o CC e o VME estiveram acima do exigido na literatura estatística (Hair et. al., 2005; Marôco, 2010), respectivamente, 0,93 e 0,74, contemplando a dimensão total do IRT, evidenciando a confiabilidade e validade convergente do construto. 
Tabela 4: Estrutura Fatorial do EIRT.

\begin{tabular}{l|cccccc}
\hline$\xi$ (construto) & $\begin{array}{c}\chi \\
\text { (variáveis) } \\
\text { [itens] }\end{array}$ & $\lambda$ & $\begin{array}{c}\varepsilon \\
\text { (erros) }\end{array}$ & CC & VME & $\begin{array}{c}\omega \\
\text { McDonald }\end{array}$ \\
\hline $\begin{array}{l}\text { Intenção } \\
\text { rotatividade }\end{array}$ & a & IRT 1 & 0,94 & 0,89 & & \\
& IRT 2 & 0,96 & 0,92 & 0,93 & 0,74 & 0,99 \\
\hline
\end{tabular}

Notas: $\lambda=$ Escores fatoriais da estrutura; $\varepsilon$ (erros) = Erros de medida da estrutura; $\chi=$ variáveis (itens); $\xi=$ construto. VME $=$ Variância Média Extraída. $\mathrm{CC}=$ Confiabilidade Composta. Fonte: Autores.

Os resultados referentes a estrutura de um único fator foram confirmados ao observar as estimativas preditivas com base na análise de regressão, pois o modelo proposto, a partir da identificação das variáveis da EIRT, revelaram uma razão critério que não apenas estava dentro do que se esperava estatisticamente, mas também que foi diferente de zero $(t>1,96, p<$ 0,05 ), com todas elas significativas (ver Tabela 5).

Na mesma Tabela 5, a partir do indicador VIF (definida em português, Inflação da Variância do Fator - IVF), com base no fator de tolerância entre as variáveis, o qual deverá apresentar escores no intervalo de 1 a 5 , para que não desqualifique no modelo empírico uma baixa estimação (Hair, Tatham, Anderson, \& Black, 2005; Marôco, 2010). Com isso, os indicadores VIF para cada item encontram-se no limite de estimação exigidos e significativos ( $p<0,01$ ), permitindo afirmar a não existência de multicolinearidade do modelo fatorial.

Tabela 5: Indicadores das estimativas preditivas da associação itens-fator da EIRT.

\begin{tabular}{ccc|ccccc}
\hline Variáveis & $<---$ & fatores & Estimativa & d.p. & $\begin{array}{c}\text { Razão } \\
\text { Critério }\end{array}$ & p-valor & VIF \\
\hline Item 1 & $<---$ & IRT & 1,000 & --- & --- & --- & 1,36 \\
Item 2 & $<---$ & IRT & 1,053 & 0,036 & 29,649 & $* * *$ & 1,25 \\
Item 3 & $<---$ & IRT & 1,050 & 0,038 & 27,445 & $* * *$ & 1,17 \\
\hline
\end{tabular}

Fonte: Autores.

Considerando esses resultados, procurou-se verificar a invariância da estrutura trifatorial, a qual pretendeu avaliar os parâmetros dos itens em função da estrutura unifatorial ajustada da mensuração da EIRT. Para isso, compararam-se os indicadores TLI e CFI relativos a estrutura do modelo a ser comprovado, para o qual espelhou-se nos mesmos indicadores achados previamente (cf. Tabela 2).

De acordo com Damásio (2013; cf. Moroco, 2010; Hair, Anderson, Tatham, \& Black, 2005), para que se possa atribuir uma invariância ao modelo estrutural pretendido, espera-se que exista uma diferença $(\Delta)<0,01$ do CFI e TLI em distintas amostras. Com isso, organizaram-se duas amostras com base na distribuição da coleta destas, uma na cidade de João Pessoa-PB e a outra em Natal-RN. Desta maneira, $\mathrm{CFI}_{\mathrm{Geral}}=1,00$ e $\mathrm{CFI}_{\mathrm{PB}}=0,99$ e $\mathrm{CFI}_{\mathrm{RN}}=0,99$ e para o TLI observou-se, $\mathrm{TLI}_{\mathrm{Geral}}=1,00$ e $\mathrm{TLI}_{\mathrm{PB}}=0,99$ e TLI $\mathrm{RN}=0,99$. Desta forma, é possivel destacar que o modelo unifatorial da EIRT é invariante e que, independente da amostra, a estrutura fatorial é a mesma.

Tendo sido corroborada a estrutura fatorial da intenção a rotatividade, procurou-se verificar a validade convergente e divergente desta medida associando ao construto suporte organizacional no trabalho (SOT) e assédio moral no trabalho (AsMT). O interesse em avaliar estas correlações se deve ao fato de que, ao compreender que o suporte organizacional trata-se de uma forma de contrato psicológico funcional, centrado em torno de expectativas de trocas e benefícios mútuos, com obrigações legais, morais e financeiras da organização com seus empregados, influenciando positivamente no desempenho e 
comprometimento (cf. Formiga, Fleury, \& Souza, 2014; Silva, 2018), é possível que este construto associe-se negativamente com o IRT; enquanto que, o AsMT, ao se referir à exposição repetitiva e prolongada dos trabalhadores as situações de humilhação, vexame e constrangimento durante a jornada de trabalho (cf. Formiga, Franco, Oliveira, Araújo, Nascimento, \& Lima, 2021), provavelmente terá influência positiva no IRT.

Desta maneira, a partir de uma correlação de Pearson entre as variáveis, observou-se que SOT relacionou negativa e significativamente com a IRT $(r=-0,41, p<0,01)$ e, positivamente com AsMT ( $r=0,46, p<0,01)$. De acordo com esses resultados é possível refletir que quanto maior a percepção do suporte organizacional e satisfação profissional, provavelmente, maiores serão as atitudes inovadoras que o funcionário possa apresentar.

A partir desses resultados, efetuou-se uma Anova one-way associada ao teste post-hoc de Scheffé, com o objetivo de avaliar as diferenças na pontuação média na variável dependente (intenção a rotatividade) versus independente (cidade [João Pessoa e Natal], tipo de empresa [pública e privada] e sexo [masculino e feminino]). Considerando essa análise, em relação ao construto em si, observou-se que em todas estas variáveis, os escore médios foram semelhantes, condição essa que sugere não existir diferença significativa, permitindo refletir que a intenção a rotatividade é a mesma, independente da variação salientada nas variáveis abordadas.

Porém, sabendo que o construto rotatividade é o somatório dos itens referentes a pensamento, planejamento e vontade, procurou-se avaliar se esses itens isoladamente influenciavam nas variáveis. Sendo assim, realizou uma Manova univariada associada ao teste post-hoc de Scheffé para cada um dos itens, observando os seguintes resultados: no item IRT 1 (Você pensa em sair da empresa onde trabalha [refere-se à avaliação do pensamento]) não houve resultado significativo; no IRT 2 (Você planeja sair da empresa onde trabalha [diz respeito à avaliação do planejamento]) observou-se resultado significativo no efeito de interação Estado (RN-PB) versus Setor de trabalho (público-privado), no qual o maior escore esteve para os trabalhadores do setor privado no $\mathrm{RN}\left(\mathrm{F}_{(2,219)}=3,49, p<0.001, \mathrm{OP}=0,65\right)$; em relação ao IRT 3 (Você tem vontade de sair da empresa onde trabalha [avaliação da volição ou vontade]), observou que as mulheres tiveram escores maiores $\left(\mathrm{F}_{(1,219)}=4,10, p<0.001, \mathrm{OP}=\right.$ $0,55)$.

\section{Discussão}

Considerando os resultados deste estudo, destaca-se que a escala de intenção a rotatividade (IRT) desenvolvida por Siqueira et al. (1997), administrada em trabalhadores de dois estados brasileiros foi confirmada. Nesta direção psicométrica, a qual poderá ser acompanhada na seção de resultados, observou-se que tanto na discriminação quanto na representatividade dos itens é possível destacar que os participantes reconheceram o referido construto no seu espaço laboral. Condição a qual foi reforçada na $\mathrm{CVR}_{\text {crítico, }}$, pois este indicador revela que o conteúdo é válido conceitualmente pelos participantes.

De forma geral, com base nas análises estatísticas clássicas referentes a condição de medida do IRT, nota-se que o conteúdo do construto apresentado é compreensivo e seguro, capaz de avaliar se o trabalhador poderá intencionar, a partir de três grandes condições - pensar, planejar e decidir - à saída ou mudança da função ou da própria organização. Com isso, é possível salientar que a intenção da rotatividade não acontece do dia para a noite, mas parece existir uma espécie de hierarquia que ocorre na organização e estruturação do pensar a respeito. Bem como, não é um fenômeno tão vazio, pois na avaliação da validade convergente e divergente nota-se que a existência de um vínculo ou apoio oferecidos pelos gestores ou pela própria organização interfere no surgimento da rotatividade.

Fato esse, que é destacado na relação negativa entre a percepção do suporte organizacional e intenção a rotatividade e que já havia sido observado no estudo de Mathieu e Zajac (1990); é bem provável que o trabalhador ao perceber, de forma prognóstica, de acordo com Oliveira-Castro, Pilati e Borges-Andrade (1999; Santos, 2012) que a organização valoriza e zela 
pelo bem-estar dos empregados, fornecendo os subsídios necessários para o desempenho das atividades laborais, a intenção a rotatividade é menor.

O que se torna diferente quando na existência de experiências negativas no ambiente de trabalho, como é no caso da identificação do assédio moral; observou-se que o assédio se relacionou positivamente com a intenção de sair da empresa, tanto no pensar quanto no planejamento e volição. Considerando este fenômeno existente nas organizações, compreendido em termos do comportamento contra a honra e liberdade, a qual poderá surgir na exposição repetitiva e prolongada a situações com características do tipo: humilhação, vexame e constrangimento no decorrer da jornada de trabalho (Formiga, Franco, Oliveira, Araújo, Nascimento, \& Lima, 2021), revelam o quanto ele é capaz de influenciar na intenção de sair da organização.

Desta maneira, destaca-se que tal construto contribui tanto para o processo de avaliação diagnóstica ou prognóstica na relação trabalhador-organização, quanto na afirmação de que o instrumento é confiável e robusto para mensurar esta situação na dinâmica laboral. Sendo assim, é possível concluir que quanto maior for o investimento da organização no suporte organizacional, menor será a intenção em sair e, por outro lado, quanto maior existir o assédio moral (seja profissional ou pessoal) no ambiente de trabalho maior será a intenção em sair da organização.

Um fato que deve ser destacado neste estudo é quanto a baixa produção de estudos sobre a validade e consistência do IRT no contexto laboral, tendo sido encontrado especificamente, apenas a de Siqueira et al. (1997) e de Silva (2018). Os primeiros autores desenvolveram a escala e apresentaram indicadores psicométricos confiáveis e, o segundo autor, confirmou os achados Siqueira et al. (1997), com indicadores muito próximos aos previamente encontrados por eles. Sendo assim, destaca-se que a escala IRT, não somente é importante para o uso das atividades do RH nas organizações, mas também é um instrumento útil para detectar situações mais complexas no ambiente de trabalho, por exemplo, sofrimento psíquico, assédio moral, comportamentos contraproducentes.

\section{Considerações Finais}

Em termos gerais, o modelo estrutural garante que a medida do construto que avalia a intenção a rotatividade, referente ao comportamento que um profissional poderá apresentar no sentido de querer, voluntariamente, desligar-se da organização à qual atua, foi comprovado, apresentando indicadores psicométricos muito seguros e aceitos pela literatura estatística (Hair, Anderson, Tatham, \& Black, 2005); destaca-se também, a invariância do IRT, pois os indicadores CFI e TLI não sofreram alteração entre as amostras dos Estados brasileiros, revelando que, independentemente da questão cultural profissional é possível mensurar a IRT na mesma direção conceitual.

Apesar desses resultados se apresentarem dentro dos critérios psicométricos abordados, sugere que, em futuros estudos, tal construto deveria se associar ao construto da cultura organizacional e do capitula psicológico positivo, tendo quanto objetivo, verificar as influencias normativas e emocionais do trabalhador na sua tomada de decisão em sair da empresa; sugere também, não menos importante, adaptar e verificar como a escala IRT se comporta em contextos culturais diferentes, seja nos estados brasileiros ou países.

Por fim, ao considerar os achados nesta pesquisa, não somente assume-se o modelo unifatorial para mensurar a IRT, mas também destaca-se alguns limites no estudo: a ausência da comparação da IRT em função do tempo de serviço e cargo ocupado na organização, não menos importante, seria útil à avaliação do nível salário e qualificação profissional; em relação aos estudos futuros, é importante uma nova aplicação do IRT em termos longitudinais, focando em variáveis da experiência profissional, formação acadêmica e nível educacional, bem como um estudo que compare trabalhadores de empresas em distintos países. 
Research, Society and Development, v. 10, n. 3, e9910313084, 2021

(CC BY 4.0) | ISSN 2525-3409 | DOI: http://dx.doi.org/10.33448/rsd-v10i3.13084

\section{Referências}

Agapito, P. R. (2012). Bem-estar no trabalho e percepção de sucesso na carreira como antecedentes de intenção de rotatividade (Dissertação de mestrado não publicada, Universidade Metodista de São Paulo, São Bernardo do Campo, SP, Brasil).

Agapito, P. R., Polizzi, F. A., \& Siqueira, M. M. M. (2015). Bem-estar no trabalho e percepção de sucesso na carreira como antecedentes de intenção de rotatividade. RAM. Revista de Administração Mackenzie, 16(6), 71-93. https://doi.org/10.1590/1678-69712015/administracao.v16n6p71-93

Albuquerque, L. G. (2002). Gestão estratégica de pessoas. In: Fleury, M. T. L. (Org.). As pessoas na organização. Editora Gente.

Associação Nacional de Pesquisa e Pós-Graduação em Psicologia - ANPEPP. (2000). Contribuições para a discussão das Resoluções CNS $n^{o}$. 196/96 e CFP $N^{o} 016 / 2000$. https://bit.ly/3i0EoQz.

Borges-Andrade, J. E., Abbad, G. S., \& Mourão, L. (2012). Modelos de avaliação e aplicação em TD\&E. In G. Abbad, L. Mourão, P. P. M. Meneses, T. Zerbini, J. E. Borges-Andrade \& R. Vilas-Boas (Orgs.), Medidas de avaliação em treinamento, desenvolvimento e educação (pp. 20-35). Porto Alegre: Artmed.

Byrne, B. M. (1989). A primer of LISREL: Basic applications and programming for confirmatory factor analytic models. Springer-Verlag.

Cançado, V. L., Medeiros, Nilcéia L., \& Jeunon, E. E. (2008). O profissional da informação: uma análise baseada no modelo de múltiplos papéis de Ulrich. Perspectivas em Ciência da Informação, 13(2), 196-218. https://doi.org/10.1590/S1413-99362008000200013

Conselho Federal de Psicologia (2000). Resolução $\mathrm{n}^{\circ}$ 016/2000. Dispõe sobre a realização de pesquisa em Psicologia com seres humanos. http://www.crpsp.org.br/portal/orientacao/resolucoes_cfp/fr_cfp_016-00.aspx. Acesso em 1 jan. 2021.

Dalfovo, O., \& Dias, D. (2009). Gestão do Capital Humano: comparação na localização de software de gestão do capital humano entre os paises Brasil, Argentina e México. Revista Brasileira de Gestão de Negócios, 11(33), 333-350. https://doi.org/10.7819/rbgn.v11i33.535

Damásio, B. F. (2012) Uso da análise fatorial exploratória em psicologia. Avaliação psicológica. 11 (2) http://pepsic.bvsalud.org/scielo.php?s cript=sci_arttext\&pid=S1677-04712012000200007

Eisenberger, R., Huntington, R., Hutchison, S., \& Sowa, D. (1986). Perceived Organizational Support. Journal of Applied Psychology, 71 (3), $500-507$.

Estivalete, V. F. B., Löbler, M. L., Andrade, T., \& Visentini, M. S. (2011). (Des) Alinhamento entre as estratégias organizacionais e as estratégias de recursos humanos: o caso de uma empresa internacionalizada. Revista de Negócios, 16 (1), 49-68.

Faissal, R., Passos, A. E. V. M., Mendonça, M. C. F. et al. (2009). Atração e seleção de pessoas. (2a ed.), Editora FGV.

Faul, F., Erdfelder, E., Lang, A. G., \& Buchner, A. (2007). G* Power 3: A flexible statistical power analysis program for the social, behavioral, and biomedical sciences. Behavior research methods, 39(2), 175-191.

Fleury, A., \& Fleury. M. T. L. (2000) Estratégias empresariais e formação de competências: um quebra-cabeça caleidoscópio da indústria brasileira. São Paulo: Ed. Atlas.

Formiga, N. S., Franco, J. B. M., Oliveira, H. C. C., Araújo, L. A. S. C., Nascimento, F. S., \& Lima, E. A. de S. A. (2021). Suporte organizacional e assédio moral no trabalho: correlatos e diferenças entre funcionários da área de saúde e educação de um munícipio do Estado da Paraíba, Brasil. Psicologia e Saúde Em Debate, 7(1), 54-76. https://doi.org/10.22289/2446-922X.V7N1A5

Formiga, N. S., Freire, B. G. de O., Azevedo, I. M., \& Faria, S. de S. (2020). Correlatos entre o suporte organizacional, capital psicológico no trabalho e expectativa de futuro: um estudo com trabalhadores brasileiros. Research, Society and Development, 9(6), e155963486. https://doi.org/10.33448/rsd-v9i6.3486

Formiga, N., Souza, M., Costa, D., Gomes, M., Fleury, L., \& Melo, G. (2015, junio 30). Comprobación empirica de una medida relacionada al excesivo consumo de alcohol en Brasileños. LIBERABIT. Revista Peruana De Psicología,21(1), 91 - 101. http://revistaliberabit.com/inde x.php/Liberabit/article/view/290

Formiga, N., Fleury, L. F. D. O., \& Souza, M. A. D. (2014). Evidências de validade da escala de percepção de suporte organizacional em funcionários de empresa pública e privada. Estudos Interdisciplinares em Psicologia, 5(2), 60-76.

Friedman, B., Hatch, J., Walker, M. D. (2000). Como atrair, gerenciar e reter capital humano-Da promessa à realidade. Futura.

Hair, J. F., Tatham, R. L., Anderson, R. E. \& Black, W. (2005). Análise multivariada de dados. Bookman.

Hom, P. (1992). A meta analytical structural equations analysis of a model of employee turnover. Journal of Applied Psychology, 77, 890-909.

Joo, B. K., \& Mclean, G. N. (2006). Best Employer Studies: A Conceptual Model from a Literature Review and a Case Study. Human Resource Development Review, 228- 257

Joreskög, K. \& Sörbom, D. (1989). LISREL 7 user's reference guide. Mooresville: Scientific Software.

Lawshe, C. A (1975) Quantitative Approach To Content Validity. Personnel Psychology, 28(4), 563-575.

Lima, E. A. S. A., Lima, H. D. S. A., \& Nilton S. Formiga (2019). Suporte organizacional e assédio moral em funcionários públicos: Um estudo correlacional em trabalhadores no sertão paraibano, Brasil. Beau Bassin, Mauritius. Novas edições Acadêmicas.

Maroco, J. (2010). Análise de equações estruturais. ReportNumber. 
Research, Society and Development, v. 10, n. 3, e9910313084, 2021

(CC BY 4.0) | ISSN 2525-3409 | DOI: http://dx.doi.org/10.33448/rsd-v10i3.13084

Mathieu, J. E., \& Zajac, D. M. (1990). A review and meta-analysis of the antecedents, correlates, and consequences of organizational commitment. Psychological Bulletin, 108(2), 171-194. https://doi.org/10.1037/0033-2909.108.2.171

Mendonça, M. C. F. (2002). Retenção de Talentos por Meio de Reconhecimento e Recompensa. Dissertação de Mestrado apresentada à Escola Brasileira de Administração Pública, Centro de Formação Acadêmica e Pesquisa, Curso de Mestrado Executivo Fundação Getúlio Vargas - FGV, São Paulo.

Mintzberg, H. and Lampel, J. (1999) Reflecting on the Strategy Process. Sloan Management Review, 40, 21-30.

Mobley, W. H. (1992). Turnover: causas, consequências e controle. Ortiz.

Oliveira, Á. F., Gomide J., Sinésio, P., Bânia V. S., \& Oliveira-Silva, L. C. (2018). Análise dos fatores organizacionais determinantes da intenção de rotatividade. Temas em Psicologia, 26(2), 1031-1042. https://dx.doi.org/10.9788/TP2018.2-18Pt

Oliveira-Castro, G. A., Pilati, R., \& Borges-Andrade, J. E. (1999). Percepção de suporte organizacional: desenvolvimento e validação de um questionário. Revista de Administração Contemporânea, 3(2), 29-51.

Pereira, G. A., Formiga, N. S., \& Estevam, I. D. (2019). Análise confirmatória da medida do suporte organizacional em enfermeiros de um hospital público de uma capital do nordeste brasileiro. Boletim - Academia Paulista de Psicologia,39(96), 59-68. http://pepsic.bvsalud.org/scielo.php?script=sci_arttext\&pid=S1415-711X2019000100007\&lng=pt\&tlng=pt.

Pontes, B. R. (1996). Planejamento, recrutamento e seleção de pessoas. (2a ed.), LTR.

Santos, P. S. (2012) Construção e validação de Escala de Estresse Organizacional. Dissertação de mestrado. Instituto de Psicologia, Universidade de Brasília.

Schwab, D. P. (1991). Contextual variables in employee performance-turnover relationships. Academy of Management Journal 34 (4), $966-975$.

Silva, D. L. (2018). Intenção de Rotatividade, Comprometimento, Percepção de Suporte e Identificação Organizacional: um Estudo na AGU. Dissertação de Mestrado, Universidade de Brasília, UnB. Brasília, DF.

Silveira, C. C. (2011). Análise de turnover na Química Brasil Ltda.

Siqueira, M. M. M. (1995). Antecedentes de comportamentos de cidadania organizacional: análise de um modelo pós-cognitivo. Tese de doutorado, Universidade de Brasília, Brasília, DF, Brasil.

Siqueira, M. M. M., Gomide, S., Jr., Moura, O. I., \& Marques, T. M. (1997). Um modelo pós-cognitivo para intenção de rotatividade: antecedentes afetivos e cognitivos. Resumo do Congresso Interamericano de Psicologia.

Siqueira, M. M. M., Gomide, S., Jr., Oliveira, A. F. \& Polizzi, A., Filho. (2014). Intenção de rotatividade. In M. M. M. Siqueira (Ed.), Medidas do comportamento organizacional: Ferramentas de diagnóstico e de gestão (pp. 209-216). Artmed.

Steil, Andrea Valéria, Penha, Mariana Mateus, \& Bonilla, Maria Alejandra Maldonado. (2016). Antecedentes da retenção de pessoas em organizações: uma revisão de literatura. Revista Psicologia Organizações e Trabalho, 16(1), 88-102. http://pepsic.bvsalud.org/scielo.php?script=sci_arttext\&pid=S1984$66572016000100008 \& \operatorname{lng}=$ pt\&tlng=pt.

Tabachnick, B. G. \& Fidell, L. S. (1996). Using multivariate statistics. Needham Heights, MA: Allyn \& Bacon.

Tamayo, A, \& Paschoal, T. (2003). A relação da motivação para o trabalho com as metas do trabalhador. RAC-Revista de Administração Contemporânea, 7(4), 33-54.

Ulrich, D. Os campeões de recursos humanos: inovando para obter os melhores resultados. Futura, 1998.

Wilson, F.; Pan, W., \& Schumsky, D. (2012). Recalculation of the critical values for Lawshe's Content Validity Ratio. Measurement and Evaluation in Counseling and Development, 45 (3), 197-210.

Yamashita, L. K. (2007). Fatores de influência na retenção de talentos em empresas de distribuição de energia elétrica: Um estudo de caso. Dissertação (mestrado). Pontifícia Universidade Católica de Minas Gerais. 